\title{
Theoretical Properties of New Error Innovation Distribution on GARCH Model
}

\author{
Olayemi Michael Sunday*, Olubiyi Adenike Oluwafunmilola \\ Statistics Department, Faculty of Science, Ekiti State University, Ado-Ekiti, Nigeria \\ Email address: \\ apostlemike2@yahoo.com (O. M. Sunday),fun2002ola@yahoo.com (O. A. Oluwafunmilola) \\ ${ }^{*}$ Corresponding author
}

\section{To cite this article:}

Olayemi Michael Sunday, Olubiyi Adenike Oluwafunmilola. Theoretical Properties of New Error Innovation Distribution on GARCH Model. American Journal of Theoretical and Applied Statistics. Vol. 10, No. 1, 2021, pp. 9-13. doi: 10.11648/j.ajtas.20211001.12

Received: December 21, 2020; Accepted: December 31, 2020; Published: January 12, 2021

\begin{abstract}
In the last decades, many error innovations have been introduced based on different modification techniques. One of the vital methods in estimating the true parameter of any volatility models is error innovation distribution, since volatility is affected by reaction from the stock market because of political recession, insecurity, constant power failure, war, political disorder, and other economic crises. In modelling of volatility in a financial investment, error innovation distribution was found advantageous. In this paper, the researcher provided a new error innovation distribution that will serve as a competitive to other existing error innovation. The theoretical properties of the standardized exponentiated Gumbel error innovation distribution is provided and the method of estimating its parameters, by maximum likelihood estimator was proposed. The exponentiated Gumbel distribution were standardized and then converted to the new error innovation through the method of transformation. The newly established error innovation which was obtained through the method of transformation in econometrics was applied on Generalized Autoregressive Conditional Heteroskedasticity (GARCH 1,1) model. For the partial derivative of the shape and volatility parameters were unable to get the exact solution of the parameters. Therefore, a method of numerical solution BFGS was applied to obtain the estimated values of the parameters.
\end{abstract}

Keyword: Exponentiated Gumbel Distribution, Error Innovation, Maximum Likelihood Estimate, Volatility and Transformation

\section{Introduction}

In the last decades, many error innovations have been introduced based on different modification techniques. One of the vital methods in estimating the true parameter of any volatility models is error innovation distribution, since volatility is affected by reaction from the stock market because of political recession, insecurity, constant power failure, war, political disorder, and other economic crises. This could trigger variation to stock prices falling yielding to high leptokurtic, therefore Standardized exponentiated Gumbel error innovation distribution was proposed by the researchers to address such reaction.

In estimating the parameters of any volatility models error distribution is one of the important methods which was why Engle [1] proposed to utilize the innovation distribution as a slip-up distribution in estimating his proposed volatility model. Error innovation distribution plays important role in estimating the parameters of the volatility models.

This study proposed an error innovation distribution with some statistical properties.

Several researchers have introduced different error innovation distributions. To assess the parameters of heteroscedastic models, numerous distributions of error innovations were proposed, ranging from normal error innovation distribution which was proposed by Engle, Bollerslev [1, 2] and host of others.

Therefore, this paper tends to proposed a more unique error innovation distribution by improving on the existing error innovation distribution via adoption of exponentiated Gumbel distribution.

The Normal distribution was originally proposed byGauss [3] and used in (1816) where the distribution follow a normal curve and later renamed after Gauss as Gaussian distribution. Engle [1] was the first to proposed error innovation distribution for modelling volatility where is model the 
inflation rate with the assumption of standard normal error innovation distribution. The model of GARCH proposed by Bollerslev [4] also accepted this normal distribution for the error innovation and used the distribution to estimate volatility models. However, due to the limitation of normal error innovation distribution such as it is leptokurtic in nature and because of this limitation it brings about inconsistent in estimation.

The student $\mathrm{t}$ distribution was discovered by William [5] where the distribution is said to have heavy tail even though it is symmetric with a bell shape like the normal distribution. Bollerslev [2] introduced Student-t error innovation distribution. The error innovation of this distribution was used to capture the limitation of the normal distribution. Bollerslev [2] was the first to model financial time series of exchange rate and stock returns on GARCH modelling using error innovation of student $t$ distribution.

Student- $t$ distribution was used in the estimation of volatility models and its captures the observed kurtosis.

Generalized Error Distribution (GED) was proposed for error Innovation by Nelson [6] in estimating EGARCH model. The GED incorporates both the normal, laplace and uniform distribution. The GED in the word of Nelson [6] was noted to be more appealing in term of fulfilling stationarity compared to that of the student-t error innovation distribution This distribution is another opposing distribution with standardized student-t distribution.

O'Hagan and Leonard [7] introduced skewed normal distribution to capture the skewness of the normal distribution while more detailed work on the distribution was given by Azzalini [8-10]. Fermandez and Steel [11] used skewed normal distribution for error innovation on volatility modelling. The skewed normal distribution is a substitute to the normal distribution and was proposed to account for asymmetric properties of error innovation distribution of assets returns. The establishment of this error innovation distribution was basically to introduced skewness parameter.

The distribution was introduced to handle the limitation of the student- $t$ distribution because the student- $t$ distribution is symmetric which can only capture symmetric returns and cannot capture asymmetric returns. Therefore, Hansen [12] filled the gap of the student-t distribution by introducing a skewed parameter to the student-t distribution called the skewed student-t distribution.

The Skewed Generalized Error Distribution proposed by Theodossiou [13] is a distinct case of skew Laplace distribution for error innovation distribution. Skewed generalized error distribution was first used for error innovation in 1998 by Theodossiou he added a skewed parameter for the generalized error distribution in order to capture the skewness of the GED and that of the skewed Laplace distribution which is a distinct case of the generalized distribution.

Samson et al. [14], modeled the volatility in Nigerian stock market using skewed error innovation distribution with the objective of determining the combination of volatility model and the skewed error distribution that best captured the dynamics in the volatility of Nigerian stock market. The study estimated the parameters of GARCH $(1,1)$, APARCH $(1,1)$, GJR-GRACH $(1,1)$, IGARCH $(1,1)$ and EGARCH $(1,1)$ using skewed normal, skewed Student-t and skewed generalized error distributions. These studies have contributed in no great measure to volatility modelling but in this paper the researchersare introducing a new error innovation distribution that will be better than the existing innovation distributionsin handling volatility modelling.

Timothy et al. [15] compared the performance of GARCH models and its extensions using five innovation distributions, normal distribution, Student-t distribution, generalized error distribution, skewed Student- $t$ and skewed generalized error distribution. Data on the daily closing prices of Zenith bank $(04 / 01 / 2007$ to $31 / 12 / 2019)$ and ETI $(04 / 01 / 2007$ to $31 / 12 / 2019$ ) were obtained from cash craft website and then converted to daily returns. The volatility models estimated were GARCH $(1,1)$, TGARCH $(1,1)$, EGARCH $(1,1)$, IGARCH $(1,1)$ and GJR- GARCH $(1,1)$. The performances of these models were compared in terms of fitness and forecasting performance based on AIC and Root Mean Square Error respectively. Result of analysis revealed that GARCH models and its extensions estimated using the skewed generalized error distribution performed better than other error innovation distributions used. The TGARCH $(1,1)$ and E-GARCH $(1,1)$ were recommended as the best model for predicting the volatility in ETI and Zenith bank stocks respectively.

\section{Methodology}

\subsection{Introduction}

In this section the researchers will be standardizing and convert an existing distribution known and called Exponentiated Gumbel Distribution introduced by Nadarajah [16] into an error innovation distribution through the method of Maximum Likelihood Estimation and then be applied to GARCH model.

Gupta [17] computed the PDF of the Exponentiated G distribution where they introduced a class of Exponentiated distribution. Gupta [17] specified the CDF and PDF as follow:

$$
F(x)=G(x)^{\alpha} ;
$$

where $u>0$ is a shape parameter and $G(x)$ is the CDF of any baseline distribution.

To obtain the PDF of the above CDF is by taking the differential equation with respect to $\mathrm{x}$ to give:

$$
f(x)=u G(x)^{\alpha-1} g(x)
$$

where $u>0$ is a shape parameter, and

$$
g(x)=\frac{d G(x)}{d x}
$$

\subsection{Exponentiated Gumbel Distribution (EGD)}

Exponentiated Gumbel Distribution was proposed by 
Nadarajah (2006). The CDF is given as:

Therefore, EGD has the density function (pdf):

$$
\begin{gathered}
F(x, \alpha, \mu, \sigma)=1-\left[1-\exp \left\{-\exp \left(-\frac{x-\mu}{\sigma}\right)\right\}\right]^{\alpha}, \alpha> \\
0,-\infty<x<\infty,-\infty<u<\infty \\
f(x, \alpha, \mu, \sigma)=\frac{\alpha}{\sigma}\left[1-\exp \left\{-\exp \left(-\frac{x-\mu}{\sigma}\right)\right\}\right]^{\alpha-1} \exp \left(-\frac{x-\mu}{\sigma}\right) \exp \left\{-\exp \left(-\frac{x-\mu}{\sigma}\right)\right\}
\end{gathered}
$$

where $\alpha$ is the shape parameter and $\sigma$ is the scale parameter.

The equation (5) above will be converted to an error innovation.

\subsection{Proposed Standardized Exponentiated Gumbel Error Innovation Distribution (SEGEID)}

In order to obtain the standardize exponentiated Gumbel Error Innovation Distribution Let $z_{t}$ be substitute in equation 5 where $x-\mu$

which now gives the standardize exponentiated Gumbel Distribution as;

$$
f\left(z_{t} ; \alpha, \sigma\right)=\frac{\alpha}{\sigma}\left[1-\exp \left\{-\exp \left(-\frac{z_{t}}{\sigma}\right)\right\}\right]^{\alpha-1} \exp \left(-\frac{z_{t}}{\sigma}\right) \exp \left\{-\exp \left(-\frac{z_{t}}{\sigma}\right)\right\},-\infty<z_{t}<\infty,
$$

The Standardized Exponentiated Gumbel distribution (SEGD) is given as the equation (6) To obtain the Error innovation of the SEDG

Let

$$
\varepsilon_{t}=z_{t} \sigma_{t}
$$

be the transformation function.

where $\varepsilon_{t}=z_{t} \sigma_{t}$ is the error of the mean equation.

Therefore, make $z_{t}$ the subject of the formula

$$
z_{t}=\frac{\varepsilon_{t}}{\sigma_{t}}
$$

Now, differentiate with respect to $\varepsilon_{t}$

$$
\frac{d z_{t}}{d \varepsilon_{t}}=\frac{1}{\sigma_{t}}
$$

Hence

$$
z_{t} \Rightarrow \frac{\varepsilon_{t}}{\sigma_{t}}=\frac{1}{\sigma_{t}}
$$

Substitute $z_{t}$ in equation (8) into equation (6)

$$
g\left(\varepsilon_{t} ; \alpha, \sigma_{t}\right)=\frac{\alpha}{\sigma_{t}}\left[1-\exp \left\{-\exp \left(\frac{\frac{\varepsilon_{t}}{\sigma_{t}}}{\sigma_{t}}\right)\right\}\right]^{\alpha-1} \exp \left(\frac{\frac{\varepsilon_{t}}{\sigma_{t}}}{\sigma_{t}}\right) \exp \left\{-\exp \left(\frac{\frac{\varepsilon_{t}}{\sigma_{t}}}{\sigma_{t}}\right)\right\}\left(\frac{1}{\left(\sigma_{t}^{2}\right)^{\frac{1}{2}}}\right)
$$

Simplest form is given as:

$$
g\left(\varepsilon_{t} ; \alpha, \sigma_{t}\right)=\frac{\alpha}{\left(\sigma_{t}^{2}\right)^{\frac{1}{2}}}\left[1-\exp \left\{-\exp \left(\frac{\varepsilon_{t}}{\sigma_{t}^{2}}\right)\right\}\right]^{\alpha-1} \exp \left(\frac{\varepsilon_{t}}{\sigma_{t}^{2}}\right) \exp \left\{-\exp \left(\frac{\varepsilon_{t}}{\sigma_{t}^{2}}\right)\right\}\left(\frac{1}{\left(\sigma_{t}^{2}\right)^{\frac{1}{2}}}\right)
$$

The above equation (12) is the Standardized Exponentiated Gumbel Error Innovation Distribution (SEGEID).

\subsection{Likelihood Function}

$$
L(\theta)=\prod_{t=1}^{n} g\left(\varepsilon_{t} ; \alpha, \sigma_{t}\right)
$$




$$
L\left(\varepsilon_{t} ; \alpha, \sigma_{t}\right)=\prod_{t=1}^{n} g\left(\varepsilon_{t} ; \theta\right)
$$

where $\theta=\left(\alpha, \sigma_{t}\right), \alpha$ is the shape parameter, $\sigma_{t}$ is the volatility models with vector parameters

$\phi=\left(\omega, \theta_{1}, \theta_{2}, \ldots, \beta_{1}, \beta_{2}, \ldots, \gamma, \delta\right)$ respectively

$$
\begin{gathered}
g\left(\varepsilon_{t} ; \alpha, \sigma_{t}\right)=\prod_{t=1}^{n}\left(\frac{\alpha}{\left(\sigma_{t}^{2}\right)^{\frac{1}{2}}}\left[1-\exp \left\{-\exp \left(\frac{\varepsilon_{t}}{\sigma_{t}^{2}}\right)\right\}\right]^{\alpha-1} \exp \left(\frac{\varepsilon_{t}}{\sigma_{t}^{2}}\right) \exp \left\{-\exp \left(\frac{\varepsilon_{t}}{\sigma_{t}^{2}}\right)\right\}\left(\frac{1}{\left(\sigma_{t}^{2}\right)^{\frac{1}{2}}}\right)\right) \\
g\left(\varepsilon_{t} ; \alpha, \sigma_{t}\right)=\alpha^{n} \prod_{t=1}^{n}\left(\frac{1}{\left(\sigma_{t}^{2}\right)^{\frac{1}{2}}}\left[1-\exp \left\{-\exp \left(\frac{\varepsilon_{t}}{\sigma_{t}^{2}}\right)\right\}\right]^{\alpha-1} \exp \left(\frac{\varepsilon_{t}}{\sigma_{t}^{2}}\right) \exp \left\{-\exp \left(\frac{\varepsilon_{t}}{\sigma_{t}^{2}}\right)\right\}\left(\frac{1}{\left(\sigma_{t}^{2}\right)^{\frac{1}{2}}}\right)\right)
\end{gathered}
$$

Taking the log likelihood function of equation (16)

$$
\begin{aligned}
L L\left(\varepsilon_{t} ; \phi\right)= & n \log (\alpha)-\frac{n}{2} \log \left(\sigma_{t}^{2}\right)+(\alpha-1) \sum_{t=1}^{n} \log \left(1-\exp \left\{-\exp \left(\frac{\varepsilon_{t}}{\sigma_{t}^{2}}\right)\right\}\right) \\
& +\sum_{t=1}^{n} \log \left(\exp \left(\frac{\varepsilon_{t}}{\sigma_{t}^{2}}\right)\right)+\sum_{t=1}^{n} \log \left(\exp \left\{-\exp \left(\frac{\varepsilon_{t}}{\sigma_{t}^{2}}\right)\right\}\right)-\frac{n}{2} \log \left(\sigma_{t}^{2}\right)
\end{aligned}
$$

where $\theta=\left(\alpha, \sigma_{t}\right), \alpha$ is the shape parameter, $\sigma_{t}^{2}$ are the itemized volatility models with vector parameters $\varnothing=\left(\omega, \theta_{1}, \theta_{2}, \ldots, \beta_{1}, \beta_{2}, \ldots, \gamma, \delta\right)$ respectively.

$$
\text { Let } \sigma_{t}^{2} \text { GARCH }(1,1) \text { model given as } \sigma_{t}^{2}=\omega+\theta_{1} \varepsilon_{t-1}^{2}+\beta_{1} \sigma_{t-1}^{2}
$$

Now,

Let Substitute equation (18) into the equation (17)general form of the log-likelihood function of SEGEID to differentiate partially with respect to the parameter below $\alpha, \omega, \theta_{1}$ and $\beta_{1}$

$$
\begin{array}{r}
L L\left(\varepsilon_{t} ; \alpha, \omega, \theta_{1}, \beta_{1}\right)=n \log (\alpha)-\frac{n}{2} \log \left(+\omega+\theta_{1} \varepsilon_{t-1}^{2}+\beta_{1} \sigma_{t-1}^{2}\right)+(\alpha-1) \sum_{t=1}^{n} \log \left[1-\exp \left\{-\exp \left(-\frac{\varepsilon_{t}}{\omega+\theta_{1} \varepsilon_{t-1}^{2}+\beta_{1} \sigma_{t-1}^{2}}\right)\right\}\right]+ \\
\sum_{t=1}^{n} \log \left\{\exp \left(-\frac{\varepsilon_{t}}{\omega+\theta_{1} \varepsilon_{t-1}^{2}+\beta_{1} \sigma_{t-1}^{2}}\right)\right\}+\sum_{t=1}^{n}\left[\exp \left\{-\exp \left(-\frac{\varepsilon_{t}}{\omega+\theta_{1} \varepsilon_{t-1}^{2}+\beta_{1} \sigma_{t-1}^{2}}\right)\right\}\right]-\frac{1}{2} n \log \left(\omega+\theta_{1} \varepsilon_{t-1}^{2}+\beta_{1} \sigma_{t-1}^{2}\right)
\end{array}
$$

Differentiating the above equation (19) partially with respect to $\alpha, \omega, \theta_{1}$ and $\beta_{1}$ then equating to zero Let,

$$
w=-\frac{\varepsilon_{t}}{\omega+\theta_{1} \varepsilon_{t-1}^{2}+\beta_{1} \sigma_{t-1}^{2}}, u=\exp \left(-\frac{\varepsilon_{t}}{\omega+\theta_{1} \varepsilon_{t-1}^{2}+\beta_{1} \sigma_{t-1}^{2}}\right)
$$

Then,

$$
\begin{gathered}
\frac{\partial l l}{\partial \alpha}=\frac{n}{\alpha}+\sum_{t=1}^{n} \log \left[1-\exp \left\{-\exp \left(-\frac{\varepsilon_{t}}{\omega+\theta_{1} \varepsilon_{t-1}^{2}+\beta_{1} \sigma_{t-1}^{2}}\right)\right\}\right]=0 \\
\frac{\partial l l}{\partial \omega}=\frac{-n}{2\left(\omega+\theta_{1} \varepsilon_{t-1}^{2}+\beta_{1} \sigma_{t-1}^{2}\right)}+(\alpha-1) \sum_{t=1}^{n}\left[\left(\frac{\exp (-u)}{1-\exp (-u)}\right)\left(\frac{\varepsilon_{t}}{\left(\omega+\theta_{1} \varepsilon_{t-1}^{2}+\beta_{1} \sigma_{t-1}^{2}\right)^{2}}\right) \exp \left(-\frac{\varepsilon_{t}}{\omega+\theta_{1} \varepsilon_{t-1}^{2}+\beta_{1} \sigma_{t-1}^{2}}\right)\right] \\
+\sum_{t=1}^{n}\left\{\left(\frac{\exp (-w)}{\exp (-\omega)}\right)\left(-\frac{\varepsilon_{t}}{\left(\omega+\theta_{1} \varepsilon_{t-1}^{2}+\beta_{1} \sigma_{t-1}^{2}\right)^{2}}\right)\right\}+\sum_{t=1}^{n}\left[(-\exp (-u))\left(\frac{\varepsilon_{t}}{\left(\omega+\theta_{1} \varepsilon_{t-1}^{2}+\beta_{1} \sigma_{t-1}^{2}\right)^{2}}\right) \exp \left(-\frac{\varepsilon_{t}}{\omega+\theta_{1} \varepsilon_{t-1}^{2}+\beta_{1} \sigma_{t-1}^{2}}\right)\right]-\frac{n}{2}\left(\frac{1}{\omega+\theta_{1} \varepsilon_{t-1}^{2}+\beta_{1} \sigma_{t-1}^{2}}\right)=0
\end{gathered}
$$




$$
\begin{array}{r}
(\alpha-1) \sum_{t=1}^{n}\left[\left(\frac{\exp (-u)}{1-\exp (-u)}\right)\left(\frac{\varepsilon_{t} \varepsilon_{t-1}^{2}}{\left(\omega+\theta_{1} \varepsilon_{t-1}^{2}+\beta_{1} \sigma_{t-1}^{2}\right)^{2}}\right) \exp \left(-\frac{\varepsilon_{t}}{\omega+\theta_{1} \varepsilon_{t-1}^{2}+\beta_{1} \sigma_{t-1}^{2}}\right)\right]+\sum_{t=1}^{n}\left\{\left(\frac{-\exp (-u)}{\exp (-u)}\right)\left(-\frac{\varepsilon_{t} \varepsilon_{t-1}^{2}}{\left(\omega+\theta_{1} \varepsilon_{t-1}^{2}+\beta_{1} \sigma_{t-1}^{2}\right)^{2}}\right)\right\}+ \\
\sum_{t=1}^{n}\left[(-\exp (-u))\left(\frac{\varepsilon_{t} \varepsilon_{t-1}^{2}}{\left(\omega+\theta_{1} \varepsilon_{t-1}^{2}+\beta_{1} \sigma_{t-1}^{2}\right)^{2}}\right) \exp \left(-\frac{\varepsilon_{t}}{\omega+\theta_{1} \varepsilon_{t-1}^{2}+\beta_{1} \sigma_{t-1}^{2}}\right)\right]-\frac{n e_{t-1}^{2}}{2}\left(\frac{1}{\omega+\theta_{1} \varepsilon_{t-1}^{2}+\beta_{1} \sigma_{t-1}^{2}}\right)=0 \quad(22) \\
\frac{\partial l l}{\partial \beta_{1}}= \\
-\frac{n \sigma_{t-1}^{2}}{2\left(\omega+\theta_{1} \varepsilon_{t-1}^{2}+\beta_{1} \sigma_{t-1}^{2}\right)}+ \\
(\alpha-1) \sum_{t=1}^{n}\left[\left(\frac{\exp (-u)}{1-\exp (-u)}\right)\left(\frac{\varepsilon_{t} \sigma_{t-1}^{2}}{\left(\omega+\theta_{1} \varepsilon_{t-1}^{2}+\beta_{1} \sigma_{t-1}^{2}\right)^{2}}\right) \exp \left(-\frac{\varepsilon_{t}}{\omega+\theta_{1} \varepsilon_{t-1}^{2}+\beta_{1} \sigma_{t-1}^{2}}\right)\right]+\sum_{t=1}^{n}\left\{\left(\frac{-\exp (-u)}{\exp (-u)}\right)\left(\frac{-\varepsilon_{t-1}}{\left(\omega+\theta_{1} \varepsilon_{t-1}^{2}+\beta_{1} \sigma_{t-1}^{2}\right)^{2}}\right)\right\}+ \\
\sum_{t=1}^{n}\left[(-\exp (-u))\left(\frac{\varepsilon_{t} \sigma_{t-1}^{2}}{\left(\omega+\theta_{1} \varepsilon_{t-1}^{2}+\beta_{1} \sigma_{t-1}^{2}\right)^{2}}\right) \exp \left(-\frac{1}{\omega+\theta_{1} \varepsilon_{t-1}^{2}+\beta_{1} \sigma_{t-1}^{2}}\right)\right]-\frac{n \sigma_{t-1}^{2}}{2}\left(\frac{1}{\omega+\theta_{1} \varepsilon_{t-1}^{2}+\beta_{1} \sigma_{t-1}^{2}}\right)=0
\end{array}
$$

\section{Discussion}

The above are the partial derivatives of the parameters for GARCH $(1,1)$ model but due to the complexity, the researcher was unable to get the exact solution of the parameters. Therefore, a method of numerical solution BFGS were applied to obtain the estimated values of the parameters.

\section{Conclusion}

An error innovation distribution called standardized exponentiated Gumbel Error Innovation Distribution (SEGEID) that will be able to model volatility such as the Generalized Autoregressive Conditional Heteroscedasticity (GARCH) effectively was proposed. The proposed model would also be able to handle outliers. It will also be effective in modelling volatility particularly in a financial investment. Finally, the SEGEID will as well capture both the symmetric and asymmetric effect in a time series data. Further research would involve comparing the proposed error innovation distribution with any of the existing innovation distribution mentioned in the literature review with the aid of real data, particularly data that exhibit extreme value such as stock data.

\section{References}

[1] Engle, R. F. (1982). Autoregressive Conditional Heteroscedasticity with Estimates of the Variance of U. K. Inflation. Econometrica, 50 (4), 987-1008.

[2] Bollerslev, T. (1987). A Conditionally Heteroskedastic Time Series Model for Speculative Prices and Rates of Return. Review of Economics and Statistics, 69 (3), 542-547.

[3] Gauss, C. F. (1809). Theoriamotvscorporvmcoelestivm in sectionibvsconicisSolemambientivm (in latin). [10] Azzalini, A. (2005). The skew-normal distribution and related multivariate families. Scandinavian Journal of statistics, 32, 159-188.

[4] Bollerslev, T. (1986). Generalized Autoregressive Conditionally Heteroskedasticity. Journal of Econometrics, 31, 307-327.
[5] William, S. G. (1908). The probable error of a mean. Biometrika, 6 (1), 1-25.

[6] Nelson, D. (1991). Conditional Heteroskedasticity in Asset Returns: A New Approach. Econometrica, 59, 349-370.

[7] O'Hagan, A., and Leonard, T. (1976). Bayes estimation subject to uncertainty about parameter constraints, Biometrika, 63, 201-2012.

[8] Azzalini, A. (1985). A class of distributions which includes the normal ones. Scandinavian Journal of statistics, 12, 171-178.

[9] Azzalini, A. (1986). Further results on a class of distributions which includes the normal ones. Statistica, 46, 199-208.

[10] Azzalini, A. (2005). The skewed-normal distribution and related multivariate families. Scandinavian Journal of Statistics, 32, 159-188.

[11] Fermandez, C., and Steel, M. F. J. (1998). On Bayesian modeling of fat tails and skewness. Journal of the American Statistical Association, 93, 359-369.

[12] Hansen, B. E. (1994). Autoregressive Conditional Density Estimation. International Economic Review, 35, 705-730.

[13] Theodossiou, P. (1998) Financial data and the skewed generalized t distribution. Management Science, 44, 16501661.

[14] Samson T. K, Onwukwe C. E and Enang E. I (2020). Modelling Volatility in Nigerian Stock Market: Evidence from Skewed Error Distributions; International Journal of Modern Mathematical Sciences, 2020, 18(1): 42-57.

[15] Timothy Kayode Samson, EkaetteInyangEnang and Christian ElenduOnwukwe (2020). Estimating the Parameters of GARCH Models and Its Extension: Comparison between Gaussian and Non-Gaussian Innovation Distributions; Covenant Journal of Physical \& Life Sciences (CJPL) Vol. 8 No. 1, June 2020 ISSN: p. 2354 - 3574 e. 2354-3485.

[16] Nadarajah, S. (2006). The exponentiated Gumbel distribution with climate application. Environmetrics, 17 (1), 13-23.

[17] Gupta, R. C., Gupta, R. D., and Gupta, P. L., (1998). Modelling failure time data by Lehman alternatives. Communications in Statistics-Theory and Methods, 27 (4), 887-904. 